\title{
Stellar Orbits in Barred Galaxies with Nuclear Rings
}

\author{
Clayton H. Heller \\ Universitäts Sternwarte, Geismarlandstraße 11, D-37083 Göttingen, \\ Germany
}

Isaac Shlosman

Department of Physics and Astronomy, University of Kentucky, Lexington, $K Y$ 40506-0055, USA

\begin{abstract}
We investigate the dynamical response of stellar orbits in a rotating barred galaxy potential to the perturbation by a nuclear gaseous ring. The change in 3D periodic orbit families is examined as the gas accumulates near the inner Lindblad resonance. It is found that the $x_{2} / x_{3}$ loop extends to higher Jacobi energy and a vertical instability strip forms in each family. These strips are connected by a symmetric/anti-symmetric pair of 2:2:1 3D orbital families. A significant distortion of the $x_{1}$ orbits is observed in the vicinity of the ring, which leads to the intersection between orbits over a large range of the Jacobi integral. We also find that a moderately elliptical ring oblique to the stellar bar produces significant phase shifts in the $x_{1}$ orbital response.
\end{abstract}

About $2 / 3$ of all disk galaxies are weakly or strongly barred (de Vaucouleurs 1963), many more are ovally distorted (Bosma 1981; Kormendy 1982) or have triaxial bulges (Kormendy 1993). Central starburst activity in these galaxies often delineates $\sim$ few $\times 100 \mathrm{pc}$ size ring-like structures of star forming regions mixed with molecular gas and dust (Buta \& Crocker 1993). Nuclear "rings" seem to be associated with inner Lindblad resonances (ILRs) (Telesco \& Decher 1988; Shlosman et al.1989; Kenney etal.1992; Athanassoula 1992; Knapen etal.1995a,b). Their intrinsic shapes vary from circular to moderately elliptical, in which case they lead stellar bars by $\sim 50-90^{\circ}$. As such, nuclear rings are moderately strong perturbations on the gravitational potential of the central galactic region, thus affecting stellar orbits and gas flow there. We analyze the main stellar orbits (in the plane and 3D) in the presence of a ring (see also Heller \& Shlosman 1995).

The galaxy model consists of the superposition of four components: disk, bulge, bar, and ring. The disk is represented by a Miyamoto-Nagai potential, the bulge by a Plummer sphere, and the bar by a triaxial Ferrers density distribution. The ring is centered in the ILR region and for the models presented here is equivalent to $\sim 10^{9} \mathrm{M}_{\odot}$ or $38 \%$ of the local mass (Model D).

The characteristic diagram for the main planar prograde periodic orbits in the inner region of the model when no ring is present (Model A) is shown in the upper frame of Figure 1. The three direct families $x_{1}, x_{2}$, and $x_{3}$ are shown along with two vertical instability strips. From the vertical instability strips bifurcate pairs of symmetric/anti-symmetric 3D orbital families, 2:2:1 (BAN/ABAN) and 


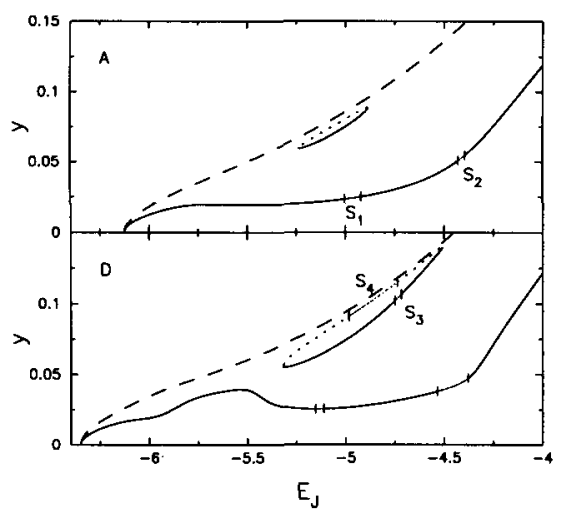

Figure 1. Characteristic diagrams of the $x_{1}, x_{2}$, and $x_{3}$ families for models without (A) and with (D) a nuclear ring. Stable sections of the characteristics are represented by solid lines while unstable are broken. Four vertical instability strips are marked. The long-dashed curve is the zero velocity curve.

2:3:1 families from $S_{1}$ and $S_{2}$, respectively. The 2:3:1 families have interesting orbital shapes that are symmetric about one vertical plane while being antisymmetric about the corresponding perpendicular vertical plane.

As the mass of the ring is increased a "bump" in the $x_{1}$ family forms and broadens at an $\mathrm{E}_{\mathbf{J}}$ below the ILR. This distortion represents a local maximum in the y-extent of the orbits, resulting in a large region of the $x_{1}$ family to have orbits that intersect with other $x_{1}$ orbits at higher $E_{\mathrm{J}}$. Such orbit intersections also occur in the $x_{2}$ family as a local maximum in orbit eccentricity develops along the sequence. Also, as the ring's mass is increased the region of stability close to the plane of the 2:2:1 symmetric family increases while the $x_{2} / x_{3}$ loop extends to higher $\mathrm{E}_{\mathbf{J}}$ and develops two regions of vertical instability. These two instability strips, one on $x_{2}$ and one on $x_{3}$, are connected by a symmetric/anti-symmetric pair of 2:2:1 families elongated perpendicular to the stellar bar. The symmetric family is stable over half of its characteristic, while the anti-symmetric family is unstable everywhere. The bottom frame of Figure 1 shows the planar characteristic diagram for the model with a circular ring and indicates the location of the $x_{2} / x_{3}$ instability strips $S_{3}$ and $S_{4}$.

In Figure $2 \mathrm{a}$ we show the phase shift or twisting of the $x_{1}$ orbital alignment in response to a moderately elliptical $(e=0.4)$ ring leading the stellar bar by 60 degrees. The change in ellipticity and position angle is given in Figure $2 b$ and is compared with models A and D. It can be seen that while the eccentricity as a function of $\mathbf{E}_{\mathrm{J}}$ is only slightly offset from the circular ring case, the position angle of the orbit semi-major axis swings from -10 to 35 degrees with respect to the bar. Note, that innermost $x_{1}$ orbits trail the bar. The interior orbits remain stable and continue to trap a significant region of phase space around them.

The main effect of the circular nuclear ring is to produce intersecting orbits over a wide range of Jacobi energies in both the $x_{1}$ and $x_{2}$ orbit families. Gas 

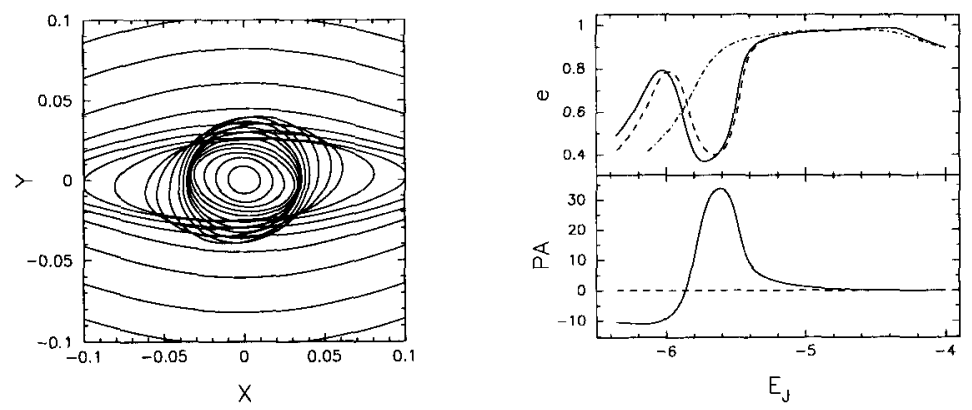

Figure 2. (a) Twisting of $x_{1}$ orbits in model with oblique elliptical ring. The ring with an ellipticity of 0.4 and semi-major axis 0.04 units is leading the bar by $60 \mathrm{deg}$. The frame is $2 \mathrm{kpc}$ on a side. (b) Eccentricity and position angle of $x_{1}$ orbits from models without ring (dot-dashed), with circular ring (dashed), and oblique elliptical ring (solid), as a function of the Jacobi energy.

on such orbits will shock and dissipate energy on a dynamical time scale. As a consequence, the gas will quickly settle down deep inside the resonance region, further enhancing the ring. The growth of the ring is limited by its self-gravity. For a non-circular ring oblique to the stellar bar and leading it, the twisting of the $x_{1}$ orbits will further enhance shocks in the gas. It is clear from Figure 2, that both trailing and leading shocks will develop.

\section{References}

Athanassoula, E. 1992, MNRAS, 259, 345

Bosma, A. 1981, ApJ, 80, 1825

Buta, R. \& Crocker, D. A. 1993, AJ, 105, 1344

de Vaucouleurs, G. 1963, ApJS, 8, 31

Heller, C. H. \& Shlosman, I. 1995, ApJ, submitted

Kenney, J. D. P., Wilson, C. D., Scoville, N. Z., Devereux, N. A. \& Young, J. S. 1992, ApJ, 395, L79

Knapen, J. H., Beckman, J. E., Shlosman, I., Peletier, R. F., Heller, C. H. \& de Jong, R. S. 1995a, ApJ, 443, L73

Knapen, J. H., Beckman, J. E., Heller, C. H., Shlosman, I., \& de Jong, R. S. 1995b, ApJ, 454623

Kormendy, J. 1982, in Morphology and Dynamics of Galaxies, L. Martinet \& M. Mayor, Sauverny: Geneva Observatory, 113

Kormendy, J. 1993, in Galactic Bulges, H. Dejonghe \& H. Habing, Dordrecht: Kluwer, 209

Shlosman, I., Frank, J., \& Begelman, M. C. 1989, Nature, 338, 45

Telesco, C. M. \& Decher, R. 1988, ApJ, 334, 573 